\title{
Non-Functioning Pancreatic Neuroendocrine Tumor
}

National Cancer Institute

\section{Source}

National Cancer Institute. Non-Functioning Pancreatic Neuroendocrine Tumor. NCI

Thesaurus. Code C45837.

\begin{abstract}
A low or intermediate grade well differentiated tumor with neuroendocrine differentiation that arises from the pancreas. It is characterized by the absence of a hormone-related clinical syndrome.
\end{abstract}

